\title{
APERIODIC INVARIANT CONTINUA FOR SURFACE HOMEOMORPHISMS
}

\author{
ANDRES KOROPECKI
}

\begin{abstract}
We prove that if a homeomorphism of a closed orientable surface $S$ has no wandering points and leaves invariant a compact, connected set $K$ which contains no periodic points, then either $K=S=\mathbb{T}^{2}$, or $K$ is the intersection of a decreasing sequence of annuli. A version for non-orientable surfaces is given.
\end{abstract}

1. Introduction. By aperiodic invariant continuum we mean a compact connected set which is invariant by some homeomorphism of a compact surface, and which contains no periodic points. We are interested in describing aperiodic invariant continua of non-wandering homeomorphisms. This type of sets appear frequently when studying generic area-preserving diffeomorphisms, due to a result of Mather Mat81, which states that for such diffeomorphisms, the boundary of certain open invariant sets (see Definition 2.1) is a finite union of aperiodic continua. Thus, having good topological information about aperiodic invariant continua is helpful to describe the dynamics of a $C^{r}$-generic area-preserving diffeomorphism. An example of this is the work of Franks and Le Calvez in [FL03] in the case that the surface is a sphere.

Our main result is the following

Theorem 1.1. Let $f: S \rightarrow S$ be a homeomorphism of a compact orientable surface such that $\Omega(f)=S$. If $K$ is an $f$-invariant continuum, then one of the following holds:

(1) $f$ has a periodic point in $K$;

(2) $K$ is annular;

(3) $K=S=\mathbb{T}^{2}$;

By annular continuum we mean an intersection of a nested sequence of topological annuli (see Definition 2.6). When $S$ is non-orientable, a version of Theorem 1.1 holds, however with two extra cases: $K$ could be a non-separating continuum in a Möbius strip, and in the case that $K=S$, the surface could be $\mathbb{T}^{2}$ or the Klein bottle (Corollary 6.1).

An important result of [FL03] states that for a generic area-preserving diffeomorphism of the sphere, the stable and unstable manifolds of hyperbolic periodic points are dense. This fact was generalized to an arbitrary surface by Xia [Xia06, and one of the main steps of his proof is obtaining a version of Theorem 1.1 which assumes generic conditions on the (area-preserving) diffeomorphism and is restricted to continua which are the closure of a particular kind of open sets. Thus Theorem 1.1 extends Xia's result to general homeomorphisms without wandering points (which includes area-preserving homeomorphisms), with no additional hypothesis on the continuum and no genericity conditions. 
A question that motivates studying aperiodic invariant continua is the following:

Question 1.2. What are the possible obstructions to the transitivity of a $C^{r}$-generic area-preserving diffeomorphism?

Bonatti and Crovisier proved in $\mathrm{BC04}$ that a $C^{1}$-generic area-preserving diffeomorphism of a compact manifold (of any dimension) is transitive. However, in dimension 2, it is known that this is not true in the $C^{r}$ topology if $r$ is large enough, because of the KAM phenomenon: There are open sets of diffeomorphisms where a $C^{r}$-generic element has an elliptic periodic point surrounded by invariant circles (see, for instance, Dou92), and this is an obstruction to transitivity. Hence the question is: is this the only possible obstruction? In other words, does the nontransitivity of a $C^{r}$-generic area-preserving diffeomorphism imply the existence of elliptic periodic points?

Studying Question 1.2 aperiodic invariant continua appear naturally as boundaries of invariant open sets. Theorem 1.1 implies that the presence of annular periodic continua is a necessary condition for the non-transitivity of a generic areapreserving diffeomorphism. In fact, a consequence of Theorem 1.1 is that the familly of aperiodic invariant continua which are minimal with respect to the property of being annular is pairwise disjoint (we call these continua frontiers, see $\mathrm{KN}$ for details). This allows a sort of decomposition of the dynamics in terms of the aperiodic invariant continua. Similar concepts appear in the work of Jäger [J09] (where the word circloid is used instead of frontier) when studying nonwandering homeomorphisms of the torus with bounded mean motion.

In $[\mathrm{KN}$, these observations play a fundamental role in the proof of the following result: for any $r \geq 1$, given a $C^{r}$-generic pair of area-preserving diffeomorphisms of a compact surface, the iterated function system (or, equivalently, the action of the semi-group) generated by them is transitive.

We should mention that the basic idea of the proof of Theorem 1.1 is inspired by the analogous result from [FL03] in the case where the surface is a sphere.

This article is organized as follows. In $\S 1-5$ we recall some background and results about ideal boundary points, continua, Lefschetz numbers and indices and we prove some elementary facts; in $\S 5$ we prove our main theorem, and a corollary about rotation numbers is mentioned; in $\S 6$ we state a version of the theorem for non-orientable surfaces, with an outline of the proof.

Acknowledgments. I am grateful to M. Nassiri for motivating this problem, as well as L. N. Carvalho, J. Franks and E. R. Pujals for useful discussions.

2. Ideal boundary, continua, and complementary domains. If $U$ is a noncompact surface, a boundary representative of $U$ is a sequence $P_{1} \supset P_{2} \supset \cdots$ of connected unbounded (i.e. not relatively compact) open sets in $U$ such that $\partial_{U} P_{n}$ is compact for each $n$ and for any compact set $K \subset U$, there is $n_{0}>0$ such that $P_{n} \cap K=\emptyset$ if $n>n_{0}$. Two boundary representatives $\left\{P_{i}\right\}$ and $\left\{P_{i}^{\prime}\right\}$ are said to be equivalent if for any $n>0$ there is $m>0$ such that $P_{m} \subset P_{n}^{\prime}$, and vice-versa. The ideal boundary of $U$ is defined as the set $\mathrm{b}_{\mathrm{I}} U$ of all equivalence classes of boundary representatives. We denote by $U^{*}$ the space $U \cup \mathrm{b}_{\mathrm{I}} U$ with the topology generated by sets of the form $V \cup V^{\prime}$, where $V$ is an open set in $U$ such that $\partial_{U} V$ is compact, and $V^{\prime}$ denotes the set of elements of $\mathrm{b}_{\mathrm{I}} U$ which have some boundary representative $\left\{P_{i}\right\}$ such that $P_{i} \subset V$ for all $i$. We call $U^{*}$ the ideal completion of $U$. Any homeomorphism $f: U \rightarrow U$ extends to a homeomorphism $f^{*}: U^{*} \rightarrow U^{*}$ 
such that $\left.f^{*}\right|_{U}=f$. If $U$ is orientable and $\mathrm{b}_{\mathrm{I}} U$ is finite, then $U^{*}$ is a compact orientable boundaryless surface. See [Ric63] and AS60] for more details.

From now on, $S$ will denote a compact orientable surface. Let $U$ be an open connected subset of $S$. For each $p^{*} \in \mathrm{b}_{\mathrm{I}} U$, we write $Z\left(p^{*}\right)$ for the set $\mathrm{cl}_{S}\left(\bigcap_{V} V \cap U\right)$ where the intersection is taken over all neighborhoods $V$ of $p^{*}$ in $U^{*}$. It is easy to see that $Z\left(p^{*}\right)$ is a compact, connected, nonempty set (see Mat82).

Definition 2.1. We say that $U \subset S$ is a complementary domain if it is a connected component of the complement of some compact connected subset of $S$.

The next proposition is a direct consequence of [Mat81, Lemma 2.3].

Proposition 2.2. If $U$ is a complementary domain in $S$, then it has finitely many ideal boundary points.

If $\mathrm{b}_{\mathrm{I}} U$ is finite, for each $p^{*} \in \mathrm{b}_{\mathrm{I}} U$ we may choose a neighborhood $V$ of $p$ such that $\bar{V}$ is homeomorphic to a closed disk, and such that $\bar{V} \cap \mathrm{b}_{\mathrm{I}} U=\{p\}$. Thus $V \backslash\{p\}$ is a topological annulus in $S$. And, unless $U$ is a topological disk, the boundary of $V$ is an essential simple closed curve in $S$. From this, we have

Proposition 2.3. If $U$ is a complementary domain in $S$, then $\mathrm{b}_{\mathrm{I}} U$ is finite, and there is a compact bordered surface $S_{U} \subset U$ such that $U \backslash S_{U}$ has finitely many connected components, each of which is homeomorphic to an open annulus.

Corollary 2.4. If $U$ is a complementary domain in $S$, then $\partial U$ has finitely many connected components.

Proof. Choose $K \subset U$ such that $U \backslash K$ is a finite union of disjoint annuli. If $A$ is a connected component of $U \backslash K$, then $\partial A \backslash U$ is connected (since it is $Z\left(p^{*}\right)$ for some $p^{*} \in \mathrm{b}_{\mathrm{I}} U$ ), and $\partial U=\bigcup_{A} \partial A \backslash U$, where the union is taken over all connected components $A$ of $U \backslash K$. Since these are finitely many, the claim follows.

We remark that the number of boundary components of $U$ may be smaller than the number of ideal boundary points, since the sets $Z\left(p^{*}\right), p^{*} \in \mathrm{b}_{\mathrm{I}} U$ need not be disjoint.

2.1. Continua. By a continuum we mean a compact connected set.

Proposition 2.5. Let $K$ be a continuum and $\mathcal{U}$ the family of all connected components of $S \backslash K$. Then all but finitely many elements of $\mathcal{U}$ are simply connected.

Proof. We consider two cases. First suppose that for some $U \in \mathcal{U}$, there is a simple closed curve $\gamma$ which is homotopically nontrivial in $U$ but trivial in $S$. Let $D$ be the topological disk bounded by $\gamma$ in $S$. Since $\gamma$ is nontrivial in $U$, there is some point of $K$ in $D$. Since $K$ is connected and $\gamma \subset S \backslash K$, it follows that $K \subset D$. Thus if $U^{\prime} \in \mathcal{U}$ and $U^{\prime} \neq U$, then $U^{\prime} \subset D$. From this we conclude that $U^{\prime}$ is simply connected. Indeed, if $\gamma^{\prime}$ is a homotopically nontrivial simple closed curve in $U^{\prime}$, then by a similar argument it bounds a disk $D^{\prime} \subset D$ which intersects $K$, so $K \subset D^{\prime}$. But this implies that $S \backslash D^{\prime} \subset U^{\prime}$ (because it is connected) so $U^{\prime}=U$, a contradiction. Therefore, all but one element of $\mathcal{U}$ are simply connected.

Now suppose that for every $U \in \mathcal{U}$, if $\gamma$ is homotopically nontrivial in $U$ then it is also homotopically nontrivial in $S$, and assume that there are infinitely many complementary domains $U_{1}, U_{2}, \ldots$ of $K$ which are not simply connected. For each 
$U_{i}$, let $\gamma_{i}$ be a simple homotopically nontrivial simple closed curve in $U_{i}$. By our assumption, $\gamma_{i}$ is also nontrivial in $S$. The curves $\left\{\gamma_{i}: i \in \mathbb{N}\right\}$ are pairwise disjoint, so there must be infinitely many of them in the same homotopy class of $S$. But if, say, $\gamma_{1}, \gamma_{2}$ and $\gamma_{3}$ are all homotopic and disjoint, there are two disjoint annuli $A_{1}$ and $A_{2}$ such that (up to reordering the indices) $\partial A_{1}=\gamma_{1} \cup \gamma_{2}$ and $\partial A_{2}=\gamma_{2} \cup \gamma_{3}$. Since the boundary of $A_{1}$ contains points of two different connected components of $S \backslash K$, it is clear that $A_{1}$ must intersect $K$. Since $K$ is connected, it follows that $K \subset A_{1}$. But with the same argument we also conclude that $K \subset A_{2}$, a contradiction. This completes the proof.

\subsection{Annular continua.}

Definition 2.6. A continuum $K \subset S$ is said to be annular if it has a neighborhood $A \subset S$ homeomorphic to an open annulus such that $A \backslash K$ has exactly two components, both homeomorphic to annuli. We call any such $A$ an annular neighborhood of $K$.

This definition is equivalent to saying that $K$ is the intersection of a sequence $\left\{A_{i}\right\}$ of closed topological annuli such that $A_{i+1}$ is an essential subset of $A_{i}$ (i.e. it separates the two boundary components of $A_{i}$ ), for each $i \in \mathbb{N}$.

3. Indices and Lefschetz number. If $f$ is a homeomorphism and $D$ is a closed topological disk without fixed points in its boundary, we denote by $\operatorname{Ind}_{f}(D)$ the fixed point index of $f$ in $D$. (see [Dol80]). If there are finitely many fixed points of $f$ in $D$, then $\operatorname{Ind}_{f}(D)$ is equal to the sum of the Lefschetz indices of these fixed points. If $D_{1}, \ldots, D_{n}$ are disjoint disks such that the set of fixed points of $f$ is contained in the interior of their union, then we have the Lefschetz formula:

$$
\sum_{i=1}^{n} \operatorname{Ind}_{f}\left(D_{i}\right)=L(f)
$$

where $L(f)$ denotes the Lefschetz number of $f$.

Lemma 3.1. Let $S$ be an orientable closed surface with Euler characteristic $\chi(S) \leq$ 0 . Then, for any homeomorphism $f: S \rightarrow S$ there is $n>0$ such that the Lefschetz number of $f^{n}$ is non-positive: $L\left(f^{n}\right) \leq 0$.

Proof. When $\chi(S)<0$, a proof can be found in Xia06. If $\chi(S)=0$, then $S \simeq \mathbb{T}^{2}$, and the automorphism induced by $f$ on $H_{1}(S, \mathbb{Q})$ can be represented by a matrix $A \in \mathrm{SL}(2, \mathbb{Z})$. It is well known that any such matrix is either periodic $\left(A^{n}=I\right.$ for some $n>0$, so $\operatorname{tr}\left(A^{n}\right)=2$ ), parabolic (and then $\operatorname{tr}\left(A^{2}\right)=2$ ) or hyperbolic (and then $\left.\operatorname{tr}\left(A^{2}\right)>2\right)$. In either case, there is $n$ such that $L\left(f^{n}\right)=2-\operatorname{tr}\left(A^{n}\right) \leq 0$.

4. Wandering points. Given a homeomorphism $f: S \rightarrow S$, we say that a nonempty open set $U$ is wandering if $f^{n}(U) \cap U=\emptyset$ for all $n>0$ (or, equivalently, for all $n \neq 0)$. We denote by $\Omega(f)$ the set of non-wandering points of $f$. That is, the (compact, invariant) set of points which have no wandering neighborhood.

Remark 4.1. We will use the following observations several times:

(1) If $\Omega(f)=S$, then $\Omega\left(f^{n}\right)=S$. To see this, given a nonempty open set $U_{0}$ we can define recursively $U_{i+1}=f^{k_{i+1}}\left(U_{i}\right) \cap U_{i}$ where $k_{i+1}>0$ is chosen such that the intersection is nonempty. Then there are integers $i_{1}<i_{2}<\cdots<i_{n}$ such that $k_{i_{1}}=k_{i_{2}}=\cdots=k_{i_{n}}(\bmod n)$, so that $k_{i_{1}}+\cdots+k_{i_{n}}=m n$ for some $m>0$, and it is easy to verify that $f^{m n}\left(U_{0}\right) \cap U_{0} \neq \emptyset$. 
(2) If $\Omega(f)=S$ and $\left\{U_{i}\right\}_{i \in \mathbb{N}}$ is a family of pairwise disjoint open sets which are permuted by $f$ (e.g. the connected components of the complement of a compact periodic set) then each $U_{i}$ is periodic for $f$.

Lemma 4.2. Let $D \subset S$ be a topological open disk and $f: \bar{D} \rightarrow \bar{D}$ a homeomorphism. Suppose that there is a neighborhood of $\partial D$ in $\bar{D}$ which does not contain the positive or the negative orbit of any wandering open set, and $f$ has no fixed points in $\partial D$. Then the index of the set of fixed points of $f$ in $D$ is 1 . In other words, there is a closed topological disk $D^{\prime}$ which contains all fixed points of $f$ in $D$, such that $\operatorname{Ind}_{f}\left(D^{\prime}\right)=1$.

Proof. Since it contains no fixed points, $\partial D$ is not reduced to a single point. By a theorem of Cartwright and Littlewood [CL51] (see also [FL03, Proposition 2.1]), the extension $\hat{f}$ of $\left.f\right|_{D}$ to the prime ends compactification $\hat{D}$ of $D$ has no fixed points in the boundary circle $\partial \hat{D}$. Thus $\hat{f}$ is orientation-preserving, and $\operatorname{Ind}_{\hat{f}}(\hat{D})=1$, and since fixed points of $\hat{f}$ are in a compact subset of $D$, we can choose a closed disk $D^{\prime} \subset D$ containing all fixed points of $\hat{f}$, so that $\operatorname{Ind}_{\hat{f}}\left(D^{\prime}\right)=\operatorname{Ind}_{\hat{f}}(\hat{D})=1$. But since $D^{\prime} \subset D$ and $\left.\hat{f}\right|_{D^{\prime}}=\left.f\right|_{D^{\prime}}$, it follows that $\operatorname{Ind}_{\hat{f}}\left(D^{\prime}\right)=\operatorname{Ind}_{f}\left(D^{\prime}\right)$ and we are done.

5. Main theorem. We begin with a brief outline of the proof. The idea is to generalize the index argument used in FL03 for the case of the sphere. However, to do that we need to modify the underlying manifold: we consider the (possibly infinitely many) connected components of $S \backslash K$. The non-wandering hypothesis guarantees that these components are permuted by $f$. Since these components are complementary domains, they have finitely many ends. Next we "remove" every nontrivial component (except for a neighborhood of its boundary), leaving a bordered submanifold $N$ of $S$ which is a neighborhood of $K$. We can modify $\left.f\right|_{N}$ obtaining a map which coincides with $f$ in a neighborhood of $K$, but which leaves the boundary of $N$ invariant. After collapsing the boundary circles of $N$ to points, we obtain a new compact surface containing $K$, and a homeomorphism which has no periodic points on $K$, and by a Lefschetz index argument we conclude that this surface can only be a sphere. From this we conclude easily that $K$ is annular.

Remark 5.1. If $K$ is an aperiodic invariant continuum and $K \neq S$, then Theorem 1.1 implies that $K$ is annular. Following [FL03, §3] (using a small annular neighborhood $A$ of $K$, and lifting $f$ to the universal covering of $A$ ) one can define the rotation set $\rho_{f}(K) \subset \mathbb{R}$ (which is defined modulo integer translations). Now, with almost no modifications, the proof of [FL03, Proposition 5.2] remains valid. Thus we obtain the following

Corollary 5.2. If $f: S \rightarrow S$ is an area preserving homeomorphism and $K \subsetneq S$ is an invariant continuum with no periodic points, then $K$ is annular, $\rho_{f}(K)$ consists of a single irrational number $\alpha$, and the rotation numbers in the prime ends from both sides of $K$ coincide (up to a sign change) with $\alpha$.

5.1. Proof of Theorem 1.1. We may assume that $f$ is orientation-preserving (otherwise consider $f^{2}$ instead of $f$ ). If $K=S$ and $f$ has no periodic points, then $S=\mathbb{T}^{2}$ by the Lefschetz theorem, and we are done. Now suppose that $f$ has no periodic points in $K$ and $K \neq S$. We need to show that $K$ is annular. 
Consider the family $\mathcal{V}$ of connected components of $S \backslash K$ which are not topological disks, which is finite by Proposition 2.5. Since open sets are nonwandering, each element of $\mathcal{V}$ is periodic by $f$. Choosing a power of $f$ instead of $f$ we may (and we do from now on) assume that each element of $\mathcal{V}$ is fixed by $f$.

Since each $V \in \mathcal{V}$ is a complementary domain, by Proposition 2.3 we can choose a compact surface with boundary $S_{V} \subset V$ such that $V \backslash S_{V}$ has finitely many components, all of which are annuli.

Given $V \in \mathcal{V}$, the ideal boundary points of $V$ are periodic by $\left(\left.f\right|_{V}\right)^{*}$, so by taking a power of $f$ instead of $f$ we may assume that they are in fact fixed. This implies that if $\gamma$ is a sufficiently small closed loop in $V$ which bounds a disk containing $p^{*}$ in $V^{*}$, then $f(\gamma)$ is homotopic to $\gamma$ in $V$ (and thus in S). Moreover, $f\left(Z\left(p^{*}\right)\right)=Z\left(p^{*}\right)$ for any $p^{*}$ in $\mathrm{b}_{\mathrm{I}} V$. Note also that $Z\left(p^{*}\right) \subset K$ for all $p^{*} \in \mathrm{b}_{\mathrm{I}} V$.

Let $A_{1}, \ldots, A_{n}$ be the connected components of $V \backslash S_{V}$. Each $A_{i}$ is a topological annulus, whose boundary in $S$ is given by a loop $\gamma_{i}$ and the continuum $Z_{i}=\bar{A}_{i} \cap K$ (which is $Z\left(p^{*}\right)$ for some $p^{*} \in V^{*}$ ). Since $f\left(Z_{i}\right)=Z_{i}$, if $\sigma_{i} \subset A_{i}$ is an essential simple closed curve close enough to $Z_{i}$, we have that $f\left(\sigma_{i}\right) \subset A_{i}$. Since $f\left(\sigma_{i}\right)$ is homotopic to $\sigma_{i}$ in $A_{i}$, there exists a homeomorphism $h_{i}: A_{i} \rightarrow A_{i}$ which maps $f\left(\sigma_{i}\right)$ to $\sigma_{i}$ and which is the identity in a neighborhood of the boundary of $A_{i}$; furthermore, we may assume that $h_{i}(x)=f^{-1}(x)$ for $x \in f\left(\sigma_{i}\right)$ (see Eps66). Extending $h_{i}$ to the identity outside $A_{i}$, and letting $\tilde{f}=h_{1} \ldots h_{n} f$, we get an orientation preserving homeomorphism such that $\tilde{f}(x)=f(x)$ for $x \in S \backslash \cup_{i} A_{i}$ and $\tilde{f}\left(\sigma_{i}\right)=\sigma_{i}$. If $\tilde{S}_{V}$ is the surface bounded by $\sigma_{1}, \ldots, \sigma_{n}$ which intersects $S_{V}$, we have that $\tilde{f}\left(\tilde{S}_{V}\right)=\tilde{S}_{V}$ and $\tilde{f}$ is the identity on the boundary of $\tilde{S}_{V}$. We do this for each $V \in \mathcal{V}$, and finally we consider the boundaryless compact surface $\tilde{S}$ obtained by collapsing each boundary circle of $S \backslash \operatorname{int} \cup_{V \in \mathcal{V}} \tilde{S}_{V}$ to a point, and the induced homeomorphism which we still call $\tilde{f}$, for which these points are fixed (see figure 1). This new surface contains $S \backslash \cup \mathcal{V}$, and $\tilde{f}$ coincides with $f$ on that set. Each $V \in \mathcal{V}$ was replaced by a (finite) union of one or more invariant topological disks, and the boundary of each of these disks is contained in $K$ (and hence, it contains no periodic points).

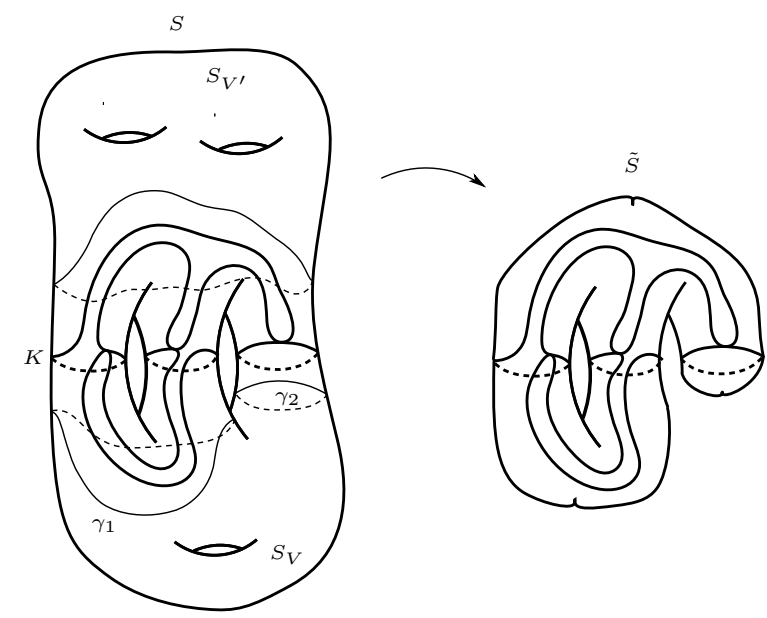

Figure 1. The complement of $K$ in $\tilde{S}$ consists of topological disks 
Since $\mathcal{V}$ consists of all components of $S \backslash K$ which are not disks, from our construction we see that all components of $\tilde{S} \backslash K$ are topological disks.

Suppose that $\chi(\tilde{S}) \leq 0$. Then by Lemma 3.1 there is $n$ such that $L\left(\tilde{f}^{n}\right) \leq 0$. Let $D$ be a connected component of $\tilde{S} \backslash K$ such that $f^{n}(D)=D$. We know that $\tilde{f}^{n}$ coincides with $f^{n}$ in a neighborhood of $\partial D \subset K$, so the fact that $f^{n}$ has no wandering points (and no fixed points in $K$ ) implies that the hypotheses of Lemma 4.2 hold. Hence, the fixed point index of $\tilde{f}^{n}$ in $D$ must be 1 (in particular, $D$ contains a fixed point). From this, it follows that there are finitely many $\tilde{f}^{n}$ invariant components in $\tilde{S} \backslash K$. In fact, if there were infinitely many, then one could find a sequence of fixed points accumulating in $K$, which contradicts the aperiodicity of $K$. Moreover, we may assume that there is at least one such component (by starting with an appropriate power of $f$ instead of $f$ ).

Since $\tilde{f}^{n}$ has no fixed points in $K$, denoting the components of $\tilde{S} \backslash K$ which are $\tilde{f}^{n}$-invariant by $D_{1}, \ldots, D_{k}$, we have from the Lefschetz formula

$$
L\left(\tilde{f}^{n}\right)=\sum_{i=1}^{k} \operatorname{Ind}_{\tilde{f}^{n}}\left(D_{i}\right)=k \geq 1,
$$

which contradicts our choice of $n$. From this we conclude that $\chi(\tilde{S})>0$, hence $\tilde{S}$ is a sphere.

But then, since $\tilde{f}$ preserves orientation, $L\left(\tilde{f}^{m}\right)=\chi(\tilde{S})=2$ for all $m$. This implies that $\tilde{S} \backslash K$ consists of exactly two components $D_{1}$ and $D_{2}$. In fact, if there were more than two such components, it would be possible to choose $m$ such that $\hat{f}^{m}$ leaves three or more of those components fixed, so that, repeating our previous argument, $L\left(\tilde{f}^{m}\right) \geq 3$, contradicting our previous claim.

Since $D_{1}$ and $D_{2}$ are topological disks, each of them is the union of an increasing sequence of closed topological disks, so that $K$ is the intersection of a decreasing sequence of annuli $\left\{A_{n}\right\}$. These annuli are eventually contained in any neighborhood of $K$, which means that, for some $n_{0},\left\{A_{n}\right\}_{n \geq n_{0}}$ is a decreasing sequence of annuli in the original surface $S$, and $\cap_{n \geq n_{0}} A_{n}=K$. Thus $K$ is annular in $S$. This completes the proof.

\section{Non-orientable case of Theorem 1.1.}

Corollary 6.1. Let $f: S \rightarrow S$ be a homeomorphism of the closed non-orientable surface $S$, such that $\Omega(f)=S$. If $K$ is an $f$-invariant continuum, then one of the following holds:

(1) $f$ has a periodic point in $K$;

(2) $K$ is annular;

(3) $K$ is the intersection of a nested sequence of Möbius strips;

(4) $K=S=$ Klein bottle.

Proof. We consider the oriented double covering $\pi: \hat{S} \rightarrow S$, and a lift $\hat{f}: \hat{S} \rightarrow \hat{S}$ of $f$. Since $f$ has no wandering points, that must be true of $\hat{f}$ as well. In fact, if $\hat{U} \subset \hat{S}$ is a sufficiently small open set, then $\pi^{-1}(\pi(\hat{U}))=\hat{U} \cup \hat{U}^{\prime}$ where the union is disjoint and $\hat{U}^{\prime}$ is homeomorphic to $\hat{U}$. If $n>0$ is such that $f^{n}(\pi(U)) \cap \pi(U) \neq \emptyset$ then either $\hat{f}^{n}(\hat{U}) \cap \hat{U} \neq \emptyset$ or $\hat{V}^{\prime}=\hat{f}^{n}(\hat{U}) \cap \hat{U}^{\prime} \neq \emptyset$. If the latter case holds, then again $\pi^{-1}\left(\pi\left(\hat{V}^{\prime}\right)\right)$ is the disjoint union of $\hat{V}^{\prime}$ and $\hat{V}$, where $\hat{V} \subset \hat{U}$, and there 
is $m>0$ such that $\hat{f}^{m}(\hat{V}) \cap \hat{V}^{\prime} \neq \emptyset$ (which implies that $\left.\hat{f}^{m+n}(\hat{U}) \cap \hat{U}\right) \neq \emptyset$ ) or $\hat{f}^{m}(\hat{V}) \cap \hat{V} \neq \emptyset$ (which implies $\hat{f}^{m}(\hat{U}) \cap \hat{U} \neq \emptyset$ ), so $\hat{U}$ is nonwandering.

Now $\pi^{-1}(K)$ consists of either a unique connected $\hat{f}$-invariant set or two copies of $K$ which are invariant by $\hat{f}$ if the lift is chosen appropriately. Let $\hat{K}$ be one of those components (or the unique component if there is only one). If $K$ has no periodic points of $f$, then $\hat{f}$ cannot have a periodic point in $\hat{K}$, because periodic points of $\hat{f}$ project to periodic points of $f$. Thus we are in the setting of Theorem 1.1, and we conclude that either $\hat{K}$ is annular or $\hat{S}=\mathbb{T}^{2}$. In the latter case, it follows that $S$ is a Klein bottle. In the former case, we have a decreasing sequence of topological annuli $\left\{\hat{A}_{i}\right\}_{i \in \mathbb{N}}$ such that $\hat{K}=\bigcap_{i} \hat{A}_{i}$. The sets $\hat{A}_{i}$ project to a decreasing sequence of neighborhoods $\left\{A_{i}\right\}_{i \in \mathbb{N}}$ of $K$, each of which is either homeomorphic to an annulus (in which case it projects injectively) or to a Möbius strip, and it is easy to see that $K=\bigcap_{i} A_{i}$. By taking a subsequence of $\left\{A_{i}\right\}_{i \in \mathbb{N}}$ if necessary, we see that either (2) or (3) must hold.

\section{REFERENCES}

[AS60] L. V. Ahlfors and L. Sario, Riemann surfaces, Princeton Univ. Press, 1960.

[BC04] C. Bonatti and S. Crovisier, Récurrence et généricité, Invent. Math. 158 (2004), no. 1, 33-104.

[CL51] M. Cartwright and J. E. Littlewood, Some fixed point theorems, Ann. of Math. 54 (1951), $1-17$.

[Dol80] A. Dold, Lectures on algebraic topology, Springer, Berlin, 1980.

[Dou92] R. Douady, Applications du théorème des tores invariants, Ph.D. thesis, Université de Paris 7, 1992, Thèse de troisiéme cycle.

[Eps66] D. B. A. Epstein, Curves on 2-manifolds and isotopies, Acta Math. 115 (1966), 83-107.

[FL03] J. Franks and P. Le Calvez, Regions of instability for non-twist maps, Ergodic Theory Dynam. Systems (2003), no. 23, 111-141.

[J09] T. Jäger, Linearisation of toral homeomorphisms, Invent. Math. 176 (2009), no. 3, 601616 .

[KN] A. Koropecki and M. Nassiri, Transitivity of generic semigroups of area-preserving surface diffeomorphisms, preprint, arXiv:0905.0305v1.

[Mat81] J. Mather, Invariant subsets of area-preserving homeomorphisms of surfaces, Advances in Math. Suppl. Studies 7B (1981), 531-561.

[Mat82] _ Topological proofs of some purely topological consequences of Caratheodory's theory of prime ends, Selected Studies (Th. M. Rassias and G. M. Rassias, eds.), NorthHolland, 1982, pp. 225-255.

[Ric63] I. Richards, On the classification of noncompact surfaces, Trans. Amer. Math. Soc. 106 (1963), no. 2, 259-269.

[Xia06] Z. Xia, Area-preserving surface diffeomorphisms, Comm. Math. Phys. 263 (2006), 723735 .

Universidade Federal Fluminense, Instituto de Matemática, Rua Mário Santos Braga S/N, 24020-140 Niteroi, RJ, BrasiL

E-mail address: koro@mat.uff.br 